\title{
Challenges in Implementation of Infection Prevention and Control Measures During the Tenth Ebola Virus Disease in Northeastern of DRC, in 2019
}

\author{
Paul Mansiangi Mankadi ${ }^{1}$, Aimee Lulebo Mampasi ${ }^{1,}$ *, Guillaume Kiyombo Mbela ${ }^{1}$, \\ Jean-Claude Makenga Bof ${ }^{2}$, Jean Nyandwe ${ }^{1}$, Jack Hyyombo Tambwe Kokolomami ${ }^{1}$ \\ ${ }^{1}$ Kinshasa School of Public Health, University of Kinshasa, Kinshasa, Democratic Republic of Congo \\ ${ }^{2}$ National Onchocerciasis Control Program, Ministry of Health, Kinshasa, Democratic Republic of Congo
}

Email address:

aimelulebo@yahoo.fr (A. L. Mampasi)

${ }^{*}$ Corresponding author

\section{To cite this article:}

Paul Mansiangi Mankadi, Aimee Lulebo Mampasi, Guillaume Kiyombo Mbela, Jean-Claude Makenga Bof, Jean Nyandwe, Jack Hyyombo Tambwe Kokolomami. Challenges in Implementation of Infection Prevention and Control Measures During the Tenth Ebola Virus Disease in Northeastern of DRC, in 2019. Central African Journal of Public Health. Vol. 6, No. 1, 2020, pp. 13-17.

doi: $10.11648 /$ j.cajph.20200601.13

Received: October 24, 2019; Accepted: December 19, 2019; Published: January 7, 2020

\begin{abstract}
Health Workers (HWs) are most likely to be infected by the Ebola virus disease (EVD) through mainly nosocomial transmission because they treat patients with EVD; this occurs through close contact with patients when infection prevention and control (IPC) measures are not strictly practiced. This study aimed to assess the level of implementation of infection prevention and control measures as well as challenges in their implementation in the northeastern region of the Democratic Republic of the Congo (DRC). A mixed study was carried out in northeastern of DRC in August 2019. In-depth interviews were conducted with $16 \mathrm{HCW}$ in front-line and with a member of the national coordination team. The quantitative part was focused on the review of the reports of the commission for the prevention and control of infections. Interviews were audiorecorded and transcribed verbatim in Swahili before being translated in French and in English. The IPC packages were implemented in the health Facilities and in the community. We noticed a high proportion of realization of the IPC planned activities. All HCWs interviewed declared to have been trained on IPC and supported with materials and equipment for implementation. Cultural and traditional views of community have been mentioned as the community related factor which impact negatively in IPC implementation by the HCWs. Non-compliance with IPC measures by HCWs has been also mentioned added with the non viability of healthcare facilities. It is urgent to improve communication of importance to comply with IPC measures in community but also among HCWs. Also, Infrastructure of Health Care Facility must be strengthened.
\end{abstract}

Keywords: HCWs, IPC, Challenges

\section{Background}

Ebola Virus Disease (EVD) become this last decade a major Public Health Concern. EVD is often a fatal illness and can cause a Case-Fatality ratio of up to $90 \%$ [1]. Currently there have been 27 EVD outbreak in Sub-Saharan African (SSA) Countries. EVD is transmitted to people from wild animals and spreads in community's trough human-to-human transmission via direct contact with blood or body fluids of a sick person or a person died from Ebola; also by objects that have been contaminated with body fluids from a person sick with Ebola or the body of a person who died from Ebola [1, $2]$.

Health Workers (HWs) are most likely to be infected by the Ebola virus disease (EVD) through mainly nosocomial transmission because they treat patients with EVD; this occurs through close contact with patients when infection prevention and control (IPC) measures are not strictly practiced [1, 3-5]. According to a systematic review performed in 2017, the proportion of HWs infected with the 
EVD varies from $2 \%$ to $100 \%$ [6].

A recommendation by the World Health Organisation (WHO) that HCWs take some hygiene measures to reduce human-to-human transmission of the EVD. These measures are: gloves and appropriate personal protective equipment that should be worn when taking care of ill patients. Regular hand washing is required after visiting patients in hospital as well as after taking care of patients at home [1]. The compliance with these measures contributes significantly to control of the EVD outbreak.

In Africa, the DRC is the country most affected by the EVD. Currently, the DRC is experiencing its tenth EVD outbreak [7]. This episode is the largest known to the country. According to the viral haemorrhagic fever (VHF) surveillance dataset, from 12 July 2018 to 17 August 2019, some 2816 cases were recorded among them 154 HWs $(151$ confirmed and three probable cases either $5.5 \%$ of HWs infected by the EVD). As a strategy to fight against the EVD outbreak, the DRC has set up nine commissions: coordination; surveillance; laboratory and research; infection prevention and control, medical care; communication and community involvement; psychosocial care; logistics and security.

Currently, there is no study describing the level of implementation and challenges in implementing infection prevention and control (IPC) measures which could explain the high frequency of the EVD among HWs. Hence, this study aimed to assess the level of implementation of infection prevention and control measures as well as challenges in their implementation.

\section{Methods}

\subsection{Study Design and Setting}

A mixed study was carried out. In-depth interviews were conducted for qualitative part; the quantitative part focused on the review of the reports of the commission for the prevention and control of infections. This study was carried out in the area of the tenth EVD outbreak, notably the Ituri and North-Kivu provinces situated in the north-eastern region of the Democratic Republic of the Congo (DRC). Data collection was performed in August 2019 more than a year after the declaration of the tenth EVD outbreak. We purposively chose three health zones (HZs) in three of the most affected districts: Katwa in Butembo city, Beni in Beni city and Mandima in Ituri Province.

\subsection{Study Population}

In-depth-interviews were carried out with $16 \mathrm{HCWs}$ in the front line and with a member of the national coordination team. Five interviews with HCWs were conducted in Katwa and Beni and six in Mandina respectively.

\subsection{Data Collection and Analysis}

A team of nine data collectors with previous experience in qualitative and quantitative data collection were recruited and trained by three supervisors, specialists in Public Health. Training was provided for three days including one day for pre-test and tool refinement. Free and informed consent was obtained from participants before commencing the interviews. Interviews were audio recorded and transcribed verbatim in Swahili before being translated into French and then into English. The qualitative study collected data on knowledge, practices and challenges in implementing IPC measures. The quantitative study collected data on level of implementation of IPC measures.

Moreover, qualitative data analysis consisted of identifying trends in the opinions expressed by the participants in relation to the topics covered. It started during data collection. Indeed, at the end of each in-depth interview, a preliminary analysis of the opinions expressed allowed interviewers to summarize the major trends related to the topics discussed. The recordings of the interviews were then transcribed using word processing software before being imported into Microsoft Excel for analysis. These transcripts were codified based on a grid of specific codes related to each topic. The analysis for the quantitative part focused on reporting the proportion of realization of the planned activities.

\section{Results}

\subsection{Quantitative Results}

\subsubsection{Package Contents for IPC}

The package for IPC was implemented at two levels, namely health facility level and community level.

The IPC package for health facility level contained twelve components, namely: 1) Creation / Revitalization of an IPC / Hygiene Committee; 2) Setting up of the Unit or zone of triage and isolation; 3) Establishment of an intra-hospital surveillance system; 4) Promoting of hand hygiene; 5) Promoting the use of personal protective equipment (PPE); 6) Waste Management; 7) Decontamination of equipment; 8) Insurance of the availability of water and energy; 9) Environmental hygiene; 10) Training of care providers; 11) Follow-up and accompaniment of the health facility; and 12) Infrastructure construction and WASH service in the health facility.

At community level the IPC package contained six components, namely: 1) Training of community leaders; 2) Community awareness of IPC activities; 3) Decontamination of households and public places; 4) Equip hygiene kits with households, schools, public places; 5) Insurance of access to water and sanitation in schools and public places; and 6) Waste Management.

\subsubsection{Level of Realization of Activities Planned}

In all 2574 health facilities that planned to benefit from package IPC, some 2560 (99.4\%) had received it. From the 2893 planned care providers, some 2669 (92.2\%) had received training. 


\subsection{Qualitative Results}

\subsubsection{Participants Characteristics}

From a total of $16 \mathrm{HWs}$ interviewed were aged 41.6 years old (7.8), males were more represented than female (11/5).

\subsubsection{Training and Availability of Materials and} Equipment for Infection Prevention and Control (IPC)

All HCWs interviewed declared to have been trained on infection prevention and control. World Health Organization Team and Health Zone members were the most cited as trainers of HCWs.

All declared to have received materials for infection prevention and control (IPC).

An HCW said, "Our health centre has received gloves, protective mask, equipment for WASH...but these materials were not enough."

\subsubsection{Knowledge on Preventive Measures for EVD}

Several hygiene measures were cited but almost all cited hand washing.

One respondent said, "At the point of entry of almost all of our health facilities you will notice the presence of the hand washing devices (large seal with $5 \%$ chlorinated water solution at 1 spoon / 201)."

A provider added, "Everything is welcome if it helps us keep our hands clean. In this way we can use water, soap, ash, hand gel, chlorine 0.5 and $0.05 \% . "$

Another said, "The first thing is to wash your hands after any action at the hospital."

Apart from hand washing, many spoke of the respect of the triage circuit and the wearing of protective equipment such as clothing / gloves before treating a patient regardless of the latter's symptomatology.

"Here in this facility, the nurses are dressed in medical clothing with boots and gloves even those who clean", said an $\mathrm{HCW}$.

A minority of HWs cited the disinfection of the under surface (soles and heels) of footwear such as boots and sandals

"Just after the washing place, one staff member with medical work wear (trousers, boots and gloves but without a mask) stands at $1 \mathrm{~m}$ with a chlorinated solution of 10 scoops / 201 of water in a container with small hose to spray the soles of the shoes of all persons entering the health facility. The inhabitants come and wash their hands themselves without being told. And also, isolation in case of suspicion of cases, good waste management, decontamination of beds after discharge of patients", said an HCP.

A minority of HWs pointed out the place of provider training in prevention.

"Our staff are well trained from triage to health care workers on prevention and protection techniques and practice them", said an HCP.

Special mention was made of the protective measures in the laboratory.

"We use the $0.5 \%$ chlorine solution, the hydro-alcoholic gel and at the exit clean water with soap. Here the protection is very strict, because it is a very dangerous environment - the waste is also the most dangerous with a dangerous evacuation", said an HCP.

Washing hands was more done with soap and with chlorinated water.

"Here at the health centre we have hand washing devices and for that people have understood and wash their hands without many problems as before", said an HCP.

The health providers respected also - the wearing of protective equipment by health workers, decontamination of beds after discharge of patients, disinfection of under surfaces (soles and heels) of shoes, sandals, isolation of suspected cases and waste management

\subsubsection{Practices of Hygiene Measures in the Health Facility and Challenges}

Hand washing with chlorinated water only. "Some people use water and soap instead of chlorinated water because for them, the smell of chlorine is not good", said an HCP.

The pre-triage is not always respected for lack of fence. Some sick guards or visitors escape this step. Isolation of the sick person is problematic because of inadequate infrastructure. The owner of the house where health centers are located sometimes refuses to isolate patients in their houses. On the other hand, some families believe that isolation of their brother or sister as being contrary to African culture. They preferred to continue in familial touching with their brothers and sitting on their hospital bed.

We kept reminding them, "When you visit a sick person neither sit on their bed nor touch anything in the hospital", said an HCP.

In this study, some people's smell perception to chlorinated water remained a constraint. However, soap is less effective against the Ebola virus. The ideal disinfectant is chlorinated water, but some people were against using it.

Regarding the community perception of health providers, some villagers believed that Ebola was a problem manufactured by health providers.

"A first case happened to our presence with headaches and fevers; he sat on the floor and did not want to be received as a suspect case because he said he has malaria not Ebola. He sat on the ground, a person from the response team tried to persuade him but without success. In the meantime, the psychologist was called", said an HCP.

Health centres without fencing prevented pre-screening of visitors and sick guards who sometimes avoided triage.

"These are adventuring their sorting circuit; let's get on the other side", said an HCP.

According to an African adage, "I am African; I will remain it all my life. You cannot prevent people from visiting or touching their sick brother or sister", said an HCP.

\subsubsection{Challenges to Implement IPC Reported by Member of Team Coordination}

Non-qualification of health care providers working in certain health facilities (They did not do any medical studies and did not receive any basic IPC training);

Non-viable, non-integrated and unregulated health training (especially those reporting nosocomial infections) with the 
consequent lack of space to install certain elements of the IPC package (triage zone, hydro-sanitary works, isolation unit, spacing) beds...);

Systematic refusal to apply SOPs and standard precautions enacted by the IPC commission in some Health Facilities (use of PPE, waste management, injection safety, hand hygiene...);

Breakdowns in the supply chain of personal protective equipment;

Failure to implement by Health Facility the follow-up plans of mentoring; and

Weak implementation of intra-hospital surveillance measures.

\section{Discussion}

According the WHO recommendations [8] this study found that, measures for EVD IPC are implemented in Northeastern of DRC to reduce human-to-human transmission of HCWs who represent a high population at risk to contract EVD and to propagate EVD in the community. The IPC activities were strongly performed as reported during the west African EVD outbreak [9].

In overall, HCWs had a good knowledge level about IPC. The essential package of IPC measures was cited by HCWs except for waste management and environment cleaning which less mentioned.

Challenges to implement IPC identified by HCWs were almost the same than mentioned by member of coordination team. These challenges are not only related to availability of resources and equipment but also to cultural and traditional views of EVD by community. This result was previously described during 2014-2015 West African outbreak [10, 11].

The non use systematically of chlorinated water by HCWs, in despite of her availability in the Healthcare facilities, was mentioned as a challenge. In literature the most challenges related to HCWs were the use systematically of equipment for individual protection because of his effect in terms of stress thermic and dehydration $[12,13]$.

The infrastructure of Healthcare facility which did not allow to HCWs to implement strictly all IPC measures like pre-triage. It is important that government takes in account this factor in making healthcare facilities more viable.

The non compliance of community at the IPC measures was mentioned as a main bottleneck to implement IPC in the Health Care Facilities. This result shows that community did not appropriate to IPC measures like observed during 20142015 West African outbreak where population had misperception on these measures and did not comply [14].

Challenges to implement IPC (lack of adequate infrastructure; cultural and behavioural factors) identified by HCWs are the major factors leading to failure to control the outbreak [15].

\section{Conclusion}

IPC activities are implemented during the tenth EVD outbreak in Northeastern of DRC, but some challenges must to be addressed for more efficacy. Then, it is urgent to improve communication of importance to comply with IPC measures in community but also among HCWs. Also Infrastructure of Health Care Facility must be strengthened. The ministry of health must pay attention to this aspect of the fight against Ebola virus disease which can compromise the success of the intervention.

\section{Limitation}

This study was limited to declaration of HCWs about of their compliance on IPC measures that could be overestimated, no observation for a long time did not perform.

\section{References}

[1] WHO. Ebola virus disease. WHO. $\mathrm{http}: / /$ www.who.int/mediacentre/factsheets/fs103/en/ (accessed August 27, 2019).

[2] Gibado S., Oladimeji A. M., Roberts A. A., Nguku P., Mwangwu I. G., Waziri N. E. et al. Public Knowledge, Perception and Source of Information on Ebola Virus Disease-Lagos, Nigeria, September 2014. Version 1. PLoS Curr. 2015 April 8; 7: ecurrents. outbreaks. 0b805cac244d700a47d6a3713ef2d6db. Published online 2015 April doi: 10.1371/currents.outbreaks.0b805cac244d700a47d6a3713ef2d6d b.

[3] World Health Organization, Health Worker Ebola Infections in Guinea, Liberia and Sierra Leone, World Health Organization, 2015.

[4] Goodfellow I, Reusken C, Koopmans M. Laboratory support during WHO. Health worker Ebola infections in Guinea, Liberia and Sierra Leone: a preliminary report 21 May 2015. WHO, Geneva 2015 .

[5] Statista. Ebola cases and deaths among health care workers due to the outbreaks in West African countries as of November 4, 2015. Disaster Med Public Health Prep. 2017 Apr; 11 (2): 262-266. doi: 10.1017/dmp.2016.103. Epub 2016 Sep 26. Accessed on February 2019.

[6] Selvaraj S. A., Lee K. E., Harrell M., Ivanov I. and Allegranzi B. Infection Rates and Risk Factors for Infection Among Health Workers During Ebola and Marburg Virus Outbreaks: A Systematic Review. The Journal of Infectious Diseases 2018; 218 (S5): S679-89.

[7] Tariq A., Roosa K., Mizumoto K., Chowella G. Assessing reporting delays and the effective reproduction number: The Ebola epidemic in DRC, May 2018-January 2019. Epidemics 26 (2019) 128-133.

[8] WHO. Infection prevention and control guidance for care of patients in health-care settings, with focus on Ebola: Interim guidance.

https://www.who.int/csr/resources/publications/ebola/filovirus _infection_control/en/ (accessed August 27, 2019).

[9] Oji MO, Haile M, Baller A, Tremblay N, Mahmoud N, Gasasira A et al. Implementing infection prevention and control capacity building strategies within the context of Ebola outbreak in a "Hard-to-Reach" area of Liberia. Pan Afr Med J. 2018; 31: 107. 
[10] Shears P., O’Dempsey T. J. D. Ebola virus disease in Africa: epidemiology and Nosocomial transmission. Journal of Hospital Infection 90 (2015) 1-9.

[11] Bausch FA, Heller O, Bengaly L, Matthey-Khouity B, Bonnabry P, Touré Yet al. Building Local Capacity in HandRub Solution Production during the 2014-2016 Ebola Outbreak Disaster: The Case of Liberia and Guinea. Prehosp Disaster Med. 2018 Dec; 33 (6): 660-667. doi: 10.1017/S1049023X18000985. Epub 2018 Nov 5.

[12] Den Boon, S., Vallenas, C., Ferri, M., \& Norris, S. L. (2018). Incorporating health workers' perspectives into a WHO guideline on personal protective equipment developed during an Ebola virus disease outbreak. F1000Research, 7, 45. https://doi.org/10.12688/f1000research.12922.2.
[13] Maynard, S. L., Kao, R., \& Craig, D. G. (2016). Impact of personal protective equipment on clinical output and perceived exertion. Journal of the Royal Army Medical Corps, 162 (3), 180-183. https://doi.org/10.1136/jramc-2015-000541.

[14] Yamanis T, Nolan E, Shepler S. Fears and Misperceptions of the Ebola Response System during the 2014-2015 Outbreak in Sierra Leone. PLoS Negl Trop Dis 201610 (10): e0005077. doi: 10.1371/journal.pntd.0005077.

[15] Coltart CEM, Lindsey B, Ghinai I, Johnson AM, Heymann DL. The Ebola outbreak, 2013-2016: old lessons for new epidemics. Phil. Trans. R. Soc. B 2017, 372: 20160297. http://dx.doi.org/10.1098/rstb.2016.0297. 